\title{
Colorectal Cancer Treatment and Survival: the Experience of Major Public Hospitals in South Australia over three Decades
}

\author{
David Roder ${ }^{1,8 *}$, Christos S Karapetis ${ }^{2}$, David Wattchow ${ }^{3}$, James Moore ${ }^{4}$, Nimit \\ Singhal $^{5}$, Rohit Joshi ${ }^{6}$, Dorothy Keefe ${ }^{7}$, Kellie Fusco ${ }^{1}$, Kate Powell ${ }^{8}$, Marion \\ Eckert $^{9}$, Timothy J Price ${ }^{10}$
}

\begin{abstract}
Background: Registry data from four major public hospitals indicate trends in clinical care and survival from colorectal cancer over three decades, from 1980 to 2010. Materials and Methods: Kaplan-Meier productlimit estimates and Cox proportional hazards models were used to investigate disease-specific survival and multiple logistic regression analyses to explore first-round treatment trends. Results: Five-year survivals increased from $48 \%$ for $1980-1986$ to $63 \%$ for $2005-2010$ diagnoses. Survival increases applied to each ACPS stage (Australian Clinico-Pathological Stage), and particularly stage C (an increase from $38 \%$ to $68 \%$ ). Risk of death from colorectal cancer halved (hazards ratio: $0.50(0.45,0.56)$ ) over the study period after adjusting for age, sex, stage, differentiation, primary sub-site, health administrative region, and measures of socioeconomic status and geographic remoteness. Decreases in stage were not observed. Survivals did not vary by sex or place of residence, suggesting reasonable equity in service access and outcomes. Of staged cases, $91 \%$ were treated surgically with lower surgical rates for older ages and more advanced stage. Proportions of surgical cases having adjuvant therapy during primary courses of treatment increased for all stages and were highest for stage $\mathrm{C}$ (an increase from $5 \%$ in 1980-1986 to $63 \%$ for 2005-2010). Radiotherapy was more common for rectal than colonic cases. Proportions of rectal cases receiving radiotherapy increased, particularly for stage $C$ where the increase was from $8 \%$ in $1980-1986$ to $60 \%$ in $2005-2010$. The percentage of stage $C$ colorectal cases less than 70 years of age having systemic therapy as part of their first treatment round increased from 3\% in 1980-1986 to 81\% by 1995-2010. Based on survey data on uptake of adjuvant therapy among those offered this care, it is likely that all these younger patients were offered systemic treatment. Conclusions: We conclude that pronounced increases in survivals from colorectal cancer have occurred at major public hospitals in South Australia due to increases in stage-specific survivals. Use of adjuvant therapies has increased and the patterns of change accord with clinical guideline recommendations. Reasons for sub-optimal use of radiotherapy for rectal cases warrant further investigation, including the potential for limited rural access to impede uptake of treatments at metropolitan-based radiotherapy centres.
\end{abstract}

Keywords: Colorectal cancer - stage - clinical care - survival trends

Asian Pac J Cancer Prev, 16 (6), 2431-2440

\section{Introduction}

Australia has an age-standardized incidence of colorectal cancer more than twice the estimated world average, but a colorectal mortality rate that exceeds the estimated world average by only $8 \%$ (Ferlay et al., 2013). Colorectal cancer is the second leading cancer in Australia both in incidence (excluding non-melanoma skin cancers) and mortality (AIHW, 2013; Ferlay et al., 2013). Only prostate cancer had a higher incidence and lung cancer a higher mortality rate (AIHW, 2013; Ferlay et al., 2013).

The age-standardized colorectal cancer incidence has been fairly stable in Australia over the past 30 years whereas death rates from this cancer have approximately halved (AIHW, 2013). Differences in these trends reflect pronounced gains in survivals, with five-year relative survivals increasing from $48 \%$ for $1982-87$ to $66 \%$ for 2006-2010 (AIHW, 2012).

${ }^{I}$ School of Population Health, University of South Australia, ${ }^{2}$ Department of Medical Oncology, Flinders Centre for Innovation in Cancer, ${ }^{3}$ Department of Surgery, Flinders Medical Centre, Flinders University, Bedford Park, ${ }^{4}$ Colorectal Surgery, ${ }^{5}$ Medical Oncology, Royal Adelaide Hospital, University of Adelaide, ${ }^{6}$ Medical Oncology, Lyell McEwen Hospital, ${ }^{7}$ University of Adelaide, ${ }^{8}$ South Australian Health and Medical Research Institute, ${ }^{9}$ Cancer Council South Australia, Eastwood, ${ }^{10}$ Medical Oncology, Queen Elizabeth Hospital, Adelaide, South Australia*For correspondence: roder@internode.on.net 
Factors responsible for national survival increases are not directly measurable due to a lack of national data on cancer stage at diagnosis, treatment and potentially other contributing factors. It has been hypothesized that survival gains may reflect both earlier diagnoses and treatment gains (Kronborg et al., 1987; U.S. Department of Health and Human Services, 1990; Hardcastle et al., 1996; Kronborg et al., 1996; Australian Health Technology Advisory Committee, 1997; NHMRC, 1999; Mandel et al., 2000; Jorgensen et al., 2002; Scholefield et al., 2002; Australian Cancer Network Colorectal Cancer Guidelines Revision Committee, 2005; Department of Health \& AIHW, 2008; Hewitson et al., 2008; Schmoll et al., 2012; Cole et al., 2013).

Randomized trials in North America and Europe showed reductions in mortality following faecal occult blood testing (FOBT) since the late 1970s (Kronborg et al., 1987; Hardcastle et al., 1996; Kronborg et al., 1996; Mandel et al., 2000; Jorgensen et al., 2002; Scholefield et al., 2002), with meta-analysis indicating an approximate $25 \%$ reduction among participants in one or more screening rounds (Hewitson et al., 2008). Australian clinicians showed a growing interest in FOBT screening since the 1980s and an increased use of endoscopic examinations, to gain earlier detection.

The Australian Health Technology Advisory Committee concluded in 1997 from trial results that population-based screening reduced colorectal cancer mortality and should be piloted (Australian Health Technology Advisory Committee, 1997). Piloting occurred in 2002-2004 and was followed by a staggered introduction of population-based screening from 2006 . Screening commenced with 55 and 65 year olds with a view to achieving biennial screening coverage of 50-74 year olds by 2020 (Department of Health \& AIHW, 2008). An interim assessment of screening impact indicated that colorectal cancers were being diagnosed at an earlier stage among people invited for screening (Cole et al., 2013).

In 1990 a U.S. National Institutes of Health Consensus Development Conference recommended adjuvant chemotherapy for TNM stage III colon and stage II and III rectal cancer, along with radiotherapy for these rectal stages (U.S. Department of Health and Human Services, 1990). Although surgery remained the mainstay of curative treatment, the importance of adjuvant therapies was underscored in guidelines issued by: $(i)$ the Australian National Health and Medical Research Council in 1999 (NHMRC, 1999); (ii) the Australian Cancer Network and Cancer Council Australia in 2005 (Australian Cancer Network Colorectal Cancer Guidelines Revision Committee, 2005); and (iii) the European Society for Medical Oncology in 2012 (Schmoll et al., 2012).

Nationally integrated data on stage and treatment are lacking, but these data exist in some clinical databases, including the South Australian Clinical Cancer Registry (SACCR) for four major public hospitals since 1980 (SACR, 1999). These hospitals serve as major referral centres in addition to providing routine care. While not population-based, their data show stage-specific survival and treatment patterns for about $40 \%$ of colorectal cancers diagnosed in South Australia (one of Australia's eight states and territories) (SACR, 1999).

The SACCR covers referral centres that encounter more advanced cancers and potentially greater comorbidity. While a national survey of colorectal cancers in 50-79 year olds indicated that approximately $24 \%$ were ACPS stage A (i.e., they had not spread beyond the muscularis propria at diagnosis) (NCCI, 2000), and a recent data-linkage study indicated a corresponding $20 \%$ of colorectal cancers were stage A among all South Australian cases aged 50-79 years (Beckmann et al., 2014a), the proportion of SACCR cases that were stage A has been lower at about 12\% (SACR, 1999). Further, 29\% of SACCR cases showed distant metastases compared with $18 \%$ of those included in the national survey and $14 \%$ of 50-79 year olds included in the South Australian linkage study (SACR, 1999; NCCI, 2000; Beckmann et al., 2014a). Five-year survivals for SACCR colorectal cases were lower during a 1991-1998 study period at 53\% (SACR, 1999), compared with a corresponding 58\% for all colorectal cases in South Australia and Australia overall (SACR, 1999; AIHW \& AACR, 2001).

With this background, SACCR data for 1980-2010 diagnoses are used now, both to show trends in riskadjusted survivals and patterns of care, and to indicate extent of concordance of treatment with guidelines. The study is descriptive and includes a further eight years of incidence data than previously reported (Luke et al., 2005).

\section{Materials and Methods}

SACCR colorectal cancer data were extracted for 1980-2010 diagnoses, with a follow-up of vital status, death dates and causes to December 31st, 2012. Operational procedures have been described previously (SACR, 1999). Ethical approval was obtained from the SA Health Human Research Ethics Committee. The SACCR operates under authorization of Section 64 of the South Australian Health Care Act (2008) primarily to support quality assurance of service delivery (NCCI, 2000). Patient consent is not legally required for use of the data for research, so long as the data are non-identifiable.

Data items covered person descriptors, dates of diagnosis, cancer sub-sites (ICD-O-3 coding or corresponding ICD-9 codes for earlier years), histology type (ICD-O-3 or SNOMED II codes for earlier years), histopathology grade, Australian Clinico-Pathological Stage (ACPS-an extension of original Dukes' staging to cover distant metastases), and date and cause of death (SACR, 1999). Pre-treatment ACPS stage was used in this study.

Death data were obtained from the SA populationbased cancer registry which extracted these data from official South Australian death files, and for deaths occurring outside of South Australia, from the National Death Index at the Australian Institute of Health and Welfare . The extent of loss to follow-up of cancer-registry cases has been checked through active tracing and found to be minimal and to have little effect on calculated survivals (Bonett et al., 1988; SACR, 1999).

Postcode of residence was used to indicate socioeconomic status quartile, using the Index of Relative 
Socioeconomic Disadvantage [note: one of the SocioEconomic Indexes for Areas (SEIFA)], geographic remoteness (classified as metropolitan, regional and remote), and Local Health Network and Medicare Local area of residence (ABS, 1998; Jong et al., 2004).

Disease-specific survival was indicated using KaplanMeier product-limit estimates, with a censoring of live cases on December 31st, 2012 (STATA, 2012). This method was preferred to relative survival because risks of deaths from competing causes could not be assumed to be equivalent to population norms (an underlying assumption for relative survival) due to the referral of high-risk cases to the tertiary referral centres covered by the SACCR (SACR, 1999).

Population-based data have shown disease-specific survival to be a good proxy for relative survival in South Australia. For example, a 1977-2003 study of colorectal cancer gave cohort relative survivals of $55 \%$ at five years, $51 \%$ at 10 years and $50 \%$ at 15 years post-diagnosis, which were similar to corresponding disease-specific survivals of $56 \%, 51 \%$ and $49 \%$ respectively(Armitage \& Berry, 1987; SACR, 2007; STATA, 2012).
Cox proportional hazards regression analyses also were used to show differences in disease-specific survival by person and tumour characteristic, both for single predictors and in a multivariable context, using the same follow-up period and censoring rules as for the KaplanMeier analyses (Armitage \& Berry, 1987; SACR, 1999; STATA, 2012). Assumptions underlying the multivariable analyses, including proportionality and lack of co-linearity, were tested and found to be met. When competing risk regression was substituted, results were similar to those of the Cox proportional hazards regression (Armitage \& Berry, 1987; STATA, 2012).

First round treatments were also recorded and patterns of care assessed by person and tumour characteristic using the Pearson chi-square or Mann-Whitney U test, depending on whether characteristics were measured on a binary, nominal or ordinal scale (Armitage \& Berry, 1987; STATA, 2012). Multiple logistic regression analysis was employed for multivariable analyses (Armitage \& Berry, 1987; STATA, 2012). Analyses were undertaken to check for confounding, effect modification and clustering by treatment centre, but did not show statistically

Table 1. \% Case Survivals from Colorectal Cancer: South Australian Major Public Hospitals, 1980-2010 Diagnoses*

\begin{tabular}{|c|c|c|c|c|c|c|c|}
\hline \multirow[b]{2}{*}{ Groups } & \multicolumn{5}{|c|}{ Period from diagnosis (yrs.) } & \multirow{2}{*}{$\begin{array}{c}\text { Hazards ratios** } \\
\text { (95\% CLs) }\end{array}$} & \multirow[t]{2}{*}{ P value** } \\
\hline & 1 & 2 & 5 & 10 & 20 & & \\
\hline All cases $(n=9098)$ & 79.8 & 69.3 & 56.1 & 50.1 & 47.1 & - & \\
\hline \multicolumn{8}{|l|}{ Age at diag. (yrs.): } \\
\hline$<40(\mathrm{n}=172)$ & 86.5 & 73.5 & 55.7 & 51.4 & 50.0 & 1.00 & $<0.001$ \\
\hline $40-49(n=502)$ & 83.0 & 70.0 & 54.8 & 49.7 & 47.5 & $1.09(0.84,1.40)$ & \\
\hline $50-59(n=1205)$ & 81.8 & 70.8 & 57.3 & 50.8 & 48.8 & $1.05(0.83,1.32)$ & \\
\hline $60-69(n=2310)$ & 84.1 & 73.7 & 59.8 & 53.8 & 50.8 & $0.97(0.77,1.22)$ & \\
\hline $70-79(n=3004)$ & 80.2 & 70.3 & 57.3 & 50.8 & 45.3 & $1.09(0.87,1.37)$ & \\
\hline $80+(n=1905)$ & 71.0 & 60.7 & 49.0 & 43.0 & 42.5 & $1.44(1.14,1.81)$ & \\
\hline \multicolumn{8}{|l|}{ Sex: } \\
\hline Male $(n=4822)$ & 80.4 & 69.8 & 55.3 & 48.6 & 46.3 & 1.00 & 0.135 \\
\hline Female $(n=4276)$ & 79.1 & 68.9 & 57.0 & 51.6 & 48.1 & $0.95(0.90,1.01)$ & \\
\hline \multicolumn{8}{|l|}{ Stage (ACPS): } \\
\hline$A(n=1186)$ & 96.8 & 95.1 & 89.3 & 83.7 & 81.4 & 1.00 & $<0.001$ \\
\hline $\mathrm{B}(\mathrm{n}=2860)$ & 93.3 & 88.2 & 75.9 & 68.9 & 64.9 & $2.16(1.82,2.56)$ & \\
\hline$C(n=2462)$ & 86.0 & 72.8 & 54.8 & 46.1 & 42.5 & $4.58(3.88,5.40)$ & \\
\hline$D(n=2067)$ & 45.1 & 24.4 & 9.9 & 7.6 & 7.2 & $19.01(16.1,22.4)$ & \\
\hline$(\mathrm{UK})(\mathrm{n}=523)$ & (74.6) & $(68.3)$ & $(59.4)$ & $(55.6)$ & $(49.4)$ & - & - \\
\hline \multicolumn{8}{|l|}{ Differentiation: } \\
\hline Well $(n=437)$ & 87.8 & 79.9 & 69.1 & 65.5 & 64.5 & 1.00 & $<0.001$ \\
\hline Moderate $(\mathrm{n}=6055)$ & 85.7 & 75.6 & 61.3 & 54.2 & 50.6 & $1.38(1.16,1.65)$ & \\
\hline Poor/undiff $(n=1904)$ & 63.2 & 50.5 & 39.5 & 35.6 & 34.5 & $2.72(2.28,3.26)$ & \\
\hline$(\mathrm{UK})(\mathrm{n}=702)$ & $(68.9)$ & $(58.5)$ & $(46.7)$ & $(43.0)$ & $(37.8)$ & - & - \\
\hline \multicolumn{8}{|l|}{ Sub-site: } \\
\hline Proximal $(\mathrm{n}=2635)$ & 76.7 & 66.1 & 56.0 & 52.0 & 50.4 & 1.00 & 0.003 \\
\hline Transverse $(n=603)$ & 79.3 & 68.7 & 58.7 & 54.2 & 49.9 & $0.94(0.82,1.08)$ & \\
\hline Distal $(\mathrm{n}=2558)$ & 80.6 & 70.6 & 57.5 & 51.4 & 47.7 & $0.96(0.88,1.04)$ & \\
\hline Other/unspec.(n-244) & 69.0 & 61.5 & 44.9 & 37.2 & 37.2 & $1.41(1.18,1.69)$ & \\
\hline Rectosigmoid $(\mathrm{n}=832)$ & 82.2 & 70.6 & 53.5 & 46.7 & 44.2 & $1.05(0.94,1.18)$ & \\
\hline Rectum $(\mathrm{n}=2226)$ & 83.0 & 72.2 & 56.2 & 48.1 & 44.7 & $0.99(0.91,1.08)$ & \\
\hline \multicolumn{8}{|l|}{ Diagnostic period: } \\
\hline $1980-86(\mathrm{n}=1133)$ & 72.4 & 60.4 & 47.6 & 42.1 & 39.1 & 1.00 & $<0.001$ \\
\hline $1987-94(n=1904)$ & 76.7 & 64.9 & 51.3 & 45.5 & 42.9 & $0.89(0.80,0.98)$ & \\
\hline $1995-99(n=1884)$ & 79.6 & 69.6 & 55.8 & 49.7 & - & $0.78(0.70,0.87)$ & \\
\hline $2000-04(n=1850)$ & 81.7 & 71.6 & 58.6 & 52.3 & - & $0.72(0.65,0.80)$ & \\
\hline $2005-10(n=2327)$ & 84.7 & 75.3 & 62.9 & - & - & $0.61(0.55,0.68)$ & \\
\hline
\end{tabular}

*Kaplan-Meier product-limit disease-specific estimates; date of censoring of live cases-Dec 31, 2012;**Derived from Cox proportional hazards regression (data in brackets excluded) 
Table 2. \% Case Survivals from Colorectal Cancer by Stage (ACPS) and Diagnostic Period: South Australian Major Public Hospitals, 1980-2010 Diagnoses*

\begin{tabular}{|c|c|c|c|c|c|c|c|c|}
\hline \multirow[b]{2}{*}{ Stage } & \multirow[b]{2}{*}{ Diagnostic period } & \multicolumn{5}{|c|}{ Period from diagnosis (yrs.) } & \multirow{2}{*}{$\begin{array}{l}\text { Hazards ratios** } \\
(95 \% \text { CLs })\end{array}$} & \multirow[t]{2}{*}{$\mathrm{P}$ value** } \\
\hline & & 1 & 2 & 5 & 10 & 20 & & \\
\hline \multirow[t]{5}{*}{ A } & $1980-86(n=118)$ & 93.0 & 91.2 & 88.3 & 78.3 & 74.5 & 1.00 & \multirow[t]{5}{*}{$<0.001$} \\
\hline & $1987-94(n=235)$ & 96.6 & 93.0 & 83.8 & 76.2 & 75.3 & $1.05(0.65,1.69)$ & \\
\hline & $1995-99(n=300)$ & 96.2 & 94.0 & 88.8 & 83.8 & - & $0.71(0.43,1.16)$ & \\
\hline & $2000-04(n=248)$ & 97.5 & 96.7 & 90.2 & 88.1 & - & $0.56(0.32,0.96)$ & \\
\hline & $2005-10(n=285)$ & 98.6 & 98.2 & 95.0 & - & - & $0.34(0.18,0.66)$ & \\
\hline \multirow[t]{5}{*}{ B } & $1980-86(n=387)$ & 90.7 & 83.3 & 67.6 & 61.2 & 56.8 & 1.00 & \multirow[t]{5}{*}{$<0.001$} \\
\hline & $1987-94(n=628)$ & 91.8 & 84.9 & 71.0 & 63.1 & 59.2 & $0.91(0.74,1.13)$ & \\
\hline & $1995-99(n=606)$ & 92.6 & 87.0 & 74.9 & 68.2 & - & $0.76(0.61,0.95)$ & \\
\hline & $2000-04(n=577)$ & 94.9 & 91.3 & 80.5 & 73.6 & - & $0.60(0.47,0.76)$ & \\
\hline & $2005-10(n=662)$ & 95.8 & 92.4 & 83.1 & - & - & $0.48(0.37,0.63)$ & \\
\hline \multirow[t]{5}{*}{$\mathrm{C}$} & $1980-86(n=313)$ & 77.1 & 57.6 & 38.2 & 32.4 & 29.0 & 1.00 & \multirow[t]{5}{*}{$<0.001$} \\
\hline & $1987-94(n=545)$ & 81.6 & 63.5 & 44.0 & 37.4 & 34.4 & $0.84(0.71,1.01)$ & \\
\hline & $1995-99(n=491)$ & 84.2 & 72.3 & 51.8 & 41.8 & - & $0.71(0.59,0.86)$ & \\
\hline & $2000-04(n=442)$ & 91.0 & 80.8 & 64.6 & 53.9 & - & $0.48(0.39,0.59)$ & \\
\hline & $2005-10(n=671)$ & 91.8 & 82.3 & 67.9 & - & - & $0.40(0.33,0.49)$ & \\
\hline \multirow[t]{5}{*}{$\mathrm{D}$} & $1980-86(n=242)$ & 28.5 & 13.8 & 6.9 & 5.8 & 5.8 & 1.00 & \multirow[t]{5}{*}{$<0.001$} \\
\hline & $1987-94(n=366)$ & 34.3 & 15.6 & 6.2 & 5.5 & 5.1 & $0.93(0.78,1.10)$ & \\
\hline & $1995-99(n=422)$ & 44.7 & 23.7 & 8.3 & 7.4 & - & $0.76(0.64,0.89)$ & \\
\hline & $2000-04(n=502)$ & 50.1 & 27.3 & 11.2 & 7.0 & - & $0.70(0.59,0.82)$ & \\
\hline & $2005-10(n=535)$ & 55.9 & 33.0 & 14.2 & - & - & $0.59(0.50,0.69)$ & \\
\hline \multirow[t]{5}{*}{ UK } & $1980-86(n=73)$ & 68.7 & 56.9 & 50.6 & 44.5 & 42.1 & 1.00 & \multirow[t]{5}{*}{0.025} \\
\hline & $1987-94(n=130)$ & 66.6 & 63.9 & 56.8 & 54.2 & 49.7 & $0.85(0.57,1.29)$ & \\
\hline & $1995-99(n=65)$ & 65.2 & 58.8 & 52.1 & 47.4 & - & $1.07(0.67,1.72)$ & \\
\hline & $2000-04(n=81)$ & 84.4 & 77.8 & 68.1 & 65.1 & - & $0.59(0.36,0.96)$ & \\
\hline & $2005-10(n=174)$ & 81.7 & 75.5 & 62.5 & - & - & $0.61(0.40,0.93)$ & \\
\hline
\end{tabular}

*Kaplan-Meier product-limit disease-specific estimates; date of censoring of live cases-Dec 31, 2012; ** Derived from Cox proportional hazards regression analyses

significant results, and so the data presented here are from conventional analyses unadjusted for such effects (STATA, 2012).

\section{Results}

\section{Survival}

Bi-variable analyses: A total of 9,098 colorectal cases were recorded with a 5-year survival of $56.1 \%$ (95\%CLs: $55.0,57.3)$ and a 10 -year survival of $50.1 \%$ (95\%CLs: 48.9, 51.2) (Table 1). Survivals were similar by sex $(\mathrm{p}=0.135)$, but differed by age category $(\mathrm{p}<0.001)$, largely due to lower survivals for patients aged 80 years and over (five-year survival of $49.0 \%$ ). No statistically significant differences in survival were found by place of residence classified by socio-economic status, remoteness, or Local Health Network or Medicare Local ( $>>0.100)$.

The more advanced the stage at diagnosis, the lower was the survival $(\mathrm{p}<0.001)$, with five-year survivals ranging from $89.3 \%$ for stage A to $9.9 \%$ for stage D. Predictably survivals reduced with decrease in cancer differentiation $(\mathrm{p}<0.001)$, from a $69.1 \%$ five-year survival for well differentiated cancers to $39.5 \%$ for poorly differentiated and undifferentiated lesions. Survivals differed by sub-site ( $\mathrm{p}=0.003)$, due to the low five-year survival of $44.9 \%$ for cases where sub-sites were unspecified/unknown compared with $56.4 \%$ for all specified colorectal sub-sites collectively (Table 1).

Survival increased over time $(\mathrm{p}<0.001)$, with the five-year survival increasing from $47.6 \%$ for $1980-86$ diagnostic years to $62.9 \%$ for $2005-2010$ (Table 1). An increase in survival was suggested over time for each stage category $(\mathrm{p}<0.001)$ (Table 2). Respective 1980-86 and 2005-2010 five-year survivals were $88.3 \%$ and $95.0 \%$ for stage A, $67.6 \%$ and $83.1 \%$ for stage B, $38.2 \%$ and $67.9 \%$ for stage C, and $6.9 \%$ and $14.2 \%$ for stage D. Cases with an unknown stage also showed a temporal increase in survival $(\mathrm{p}=0.025)$.

Multi-variable analyses: Multivariable analysis results (Table 3) confirm that risk of death from colorectal cancer varied with: (i) Age at diagnosis, with hazards ratios (95\% confidence limits) increasing with age to 1.44 $(1.14,1.80)$ for $70-79$ years and $2.13(1.69,2.69)$ for 80 years and over, compared with the reference category of under 40 years; (ii) Stage, with hazard ratios increasing with more advanced stage to $19.1(16.2,22.6)$ for stage D compared with the stage A reference category; (iii) Differentiation, with a hazards ratio of $1.83(1.53,2.20)$ for poorly differentiated and undifferentiated cancers compared with the well-differentiated reference category; (iv) Sub-site, with a hazards ratio of $1.11(1.01,1.21)$ for rectal compared with the proximal cancer reference subsite; (v) Diagnostic year, with hazards ratios decreasing from the $1980-86$ reference period to $0.50(0.45,0.56)$ for 2005-2010.

As for the unadjusted analyses, hazards ratios did not vary significantly by sex or place of residence classified by socio-economic status, remoteness, Local Health Network or Medicare Local ( $\mathrm{p}>0.100$ ).

When the multivariable model was run separately for each stage and for colon and rectum separately, adjusting for age, sex, differentiation, sub-site, and residential 
area socio-economic status, remoteness, Local Health Network and Medicare Local, decreases in hazards ratios were observed in the later diagnostic years (Table 4). Compared with 1980-86, hazards ratios for 2005-2010 were as follows: (i) Colorectal cancers: $0.31(0.16,0.62)$

Table 3. Hazards Ratios (95\% CLs) for Death from Colorectal Cancer: South Australian Major Public Hospitals, 1980-2010 Diagnoses*

\begin{tabular}{|c|c|c|}
\hline \multicolumn{3}{|c|}{ Hazards ratios } \\
\hline \multirow{6}{*}{$\begin{array}{c}\text { Age at diagnosis } \\
\text { (yrs): }\end{array}$} & $<40(\mathrm{n}=172)$ & 1.00 \\
\hline & $40-49(n=502)$ & $1.04(0.81,1.34)$ \\
\hline & $50-59(n=1205)$ & $1.11(0.88,1.41)$ \\
\hline & $60-69(n=2310)$ & $1.17(0.93,1.47)$ \\
\hline & $70-79(n=3004)$ & $1.44(1.14,1.80)$ \\
\hline & $80+(n=1905)$ & $2.13(1.69,2.69)$ \\
\hline \multirow[t]{2}{*}{ Sex: } & Male $(n=4822)$ & 1.00 \\
\hline & Female $(n=4276)$ & $0.96(0.90,1.02)$ \\
\hline \multirow[t]{5}{*}{ Stage (ACPS): } & $\mathrm{A}(\mathrm{n}=1186)$ & 1.00 \\
\hline & $B(n=2860)$ & $2.05(1.73,2.43)$ \\
\hline & $C(n=2462)$ & $4.45(3.77,5.25)$ \\
\hline & $D(n=2067)$ & $19.1(16.2,22.6)$ \\
\hline & UK (n=523) & $3.65(2.96,4.50)$ \\
\hline \multirow[t]{4}{*}{ Differentiation: } & Well $(n=437)$ & 1.00 \\
\hline & Moderate $(\mathrm{n}=6055)$ & $1.18(0.99,1.41)$ \\
\hline & Poor/ undiff. $(n=1904)$ & $1.83(1.53,2.20)$ \\
\hline & $\mathrm{UK}(\mathrm{n}=702)$ & $1.53(1.25,1.88)$ \\
\hline \multirow[t]{6}{*}{ Sub-site: } & Proximal $(n=2635)$ & 1.00 \\
\hline & Transverse $(n=603)$ & $0.91(0.79,1.05)$ \\
\hline & Distal $(n=2558)$ & $1.02(0.93,1.11)$ \\
\hline & Other/unspec. $(n=244)$ & $1.02(0.93,1.11)$ \\
\hline & Rectosigmoid $(n=832)$ & $1.05(0.94,1.18)$ \\
\hline & Rectum $(n=2226)$ & $1.11(1.01,1.21)$ \\
\hline \multirow[t]{5}{*}{ Diagnostic period: } & $1980-86(n=1133)$ & 1.00 \\
\hline & $1987-94(n=1904)$ & $0.90(0.82,1.00)$ \\
\hline & $1995-99(n=1884)$ & $0.76(0.69,0.85)$ \\
\hline & $2000-04(n=1850)$ & $0.59(0.53,0.66)$ \\
\hline & $2005-10(n=2327)$ & $0.50(0.45,0.56)$ \\
\hline
\end{tabular}

*Derived from Cox proportional hazards regression, adjusted for nonsignificant ( $\mathrm{p}>0.05$ ) associations with: socio-economic (SEIFA) quartile; remoteness of residence; Local Health Network; Medicare Local (see text). Date of censoring of live cases-Dec 31, 2012 for stage A; $0.45(0.34,0.58)$ for stage B; 0.38 (0.31, $0.47)$ for stage $C$; and $0.61(0.52,0.73)$ for stage $D$; (ii) Colon cancers: $0.46(0.15,1.38)$ for stage A; $0.44(0.32$, $0.61)$ for stage $\mathrm{B} ; 0.39(0.30,0.50)$ for stage $\mathrm{C}$; and 0.59 $(0.48,0.72)$ for stage $\mathrm{D}$; (iii) Rectal cancers: $0.26(0.10$, $0.66)$ for stage $\mathrm{A} ; 0.44(0.27,0.73)$ for stage $\mathrm{B} ; 0.38(0.27$, $0.55)$ for stage $C$; and $0.66(0.47,0.92)$ for stage $D$. By comparison, consistent time trends were not observed for cases of unknown stage (Table 4).

\section{Treatment Surgery}

Bi-variable analyses: Surgery details were recorded for 9,034 cases. Of the 8,615 with a recorded stage, $90.5 \%$ $(n=7,800)$ were treated surgically, with this proportion decreasing marginally with more advanced stage from $96.7 \%$ for stage A to $96.3 \%$ for stage B and $94.8 \%$ for stage $\mathrm{C}$, and to $73.9 \%$ for stage $\mathrm{D}(\mathrm{p}<0.001)$. Of the 419 cases without a recorded stage, only $264(63.0 \%)$ had colorectal surgery.

Multivariable analyses: Multiple logistic regression analysis indicated that predictors of surgical treatment were: $(i)$ Younger age at diagnosis, with the relative odds (95\% confidence limits) of surgery reducing with increasing age to $0.46(0.26,0.82)$ for the age 80 years or more compared with the reference category of under 40 years; ( $i i)$ Less advanced stage, with stage D having a relative odds (95\% confidence limits) of surgery of 0.10 $(0.07,0.14)$ compared with the stage A reference category; (iii) Sub-site of cancer, with lower relative odds of surgery for distal colonic lesions at $0.80(0.65,0.98)$ and rectal lesions at $0.59(0.48,0.73)$ compared with the proximal colonic reference category.

For colon cases, the most common surgical procedures were right hemicolectomies $(54.0 \%)$ followed by sigmoid colectomies $(27.0 \%)$, left hemicolectomies $(8.3 \%)$, partial colectomies $(2.3 \%)$, total colectomies $(2.3 \%)$ and other or unknown procedures $(6.1 \%)$. The distribution varied by period $(\mathrm{p}<0.001)$, with the proportion that were: (1) right

Table 4. Trends in Hazards Ratios (95\% CLs) for Death from Colorectal Cancer: South Australian Major Public Hospitals, 1980-2010 Diagnoses* - 15 Multivariable Analyses

\begin{tabular}{llccccc}
\hline \multirow{2}{*}{ Site } & Stage (ACPS) & $1980-86$ & $1987-94$ & Period from diagnosis (yrs.) & 2000-04 & $2005-10$ \\
\hline Colorectal & A (n=1186) & 1.00 & $0.97(0.59,1.59)$ & $0.63(0.38,1.07)$ & $0.48(0.27,0.86)$ & $0.31(0.16,0.62)$ \\
& B (n=2860) & 1.00 & $0.90(0.73,1.12)$ & $0.73(0.58,0.91)$ & $0.56(0.44,0.71)$ & $0.45(0.34,0.58)$ \\
& C (n=2462) & 1.00 & $0.86(0.72,1.03)$ & $0.73(0.60,0.88)$ & $0.48(0.39,0.59)$ & $0.38(0.31,0.47)$ \\
& D (n=2067) & 1.00 & $0.92(0.77,1.09)$ & $0.81(0.68,0.96)$ & $0.71(0.59,0.84)$ & $0.61(0.52,0.73)$ \\
Colon & UK (n=523) & 1.00 & $0.93(0.60,1.42)$ & $1.31(0.79,2.18)$ & $0.92(0.54,1.58)$ & $1.03(0.63,1.68)$ \\
& A (n=665) & 1.00 & $1.38(0.56,3.36)$ & $0.77(0.30,1.95)$ & $0.68(0.25,1.83)$ & $0.46(0.15,1.38)$ \\
& B (n=2049) & 1.00 & $0.81(0.62,1.06)$ & $0.65(0.49,0.86)$ & $0.50(0.37,0.67)$ & $0.44(0.32,0.61)$ \\
& C (n=1591) & 1.00 & $0.84(0.67,1.06)$ & $0.71(0.56,0.89)$ & $0.47(0.36,0.61)$ & $0.39(0.30,0.50)$ \\
\multirow{5}{*}{ Rectum } & D (n=1409) & 1.00 & $0.85(0.69,1.05)$ & $0.80(0.65,0.98)$ & $0.74(0.61,0.91)$ & $0.59(0.48,0.72)$ \\
& UK (n=326) & 1.00 & $0.65(0.37,1.14)$ & $0.76(0.38,1.51)$ & $0.38(0.18,0.91)$ & $0.57(0.27,1.19)$ \\
& A (n=521) & 1.00 & $0.90(0.48,1.69)$ & $0.61(0.31,1.19)$ & $0.43(0.21,0.91)$ & $0.26(0.10,0.66)$ \\
& B (n=811) & 1.00 & $1.06(0.73,1.54)$ & $0.89(0.60,1.32)$ & $0.68(0.45,1.05)$ & $0.44(0.27,0.73)$ \\
& C (n=871) & 1.00 & $0.99(0.72,1.36)$ & $0.85(0.61,1.17)$ & $0.53(0.38,0.76)$ & $0.38(0.27,0.55)$ \\
& D (n=658) & 1.00 & $1.16(0.83,1.62)$ & $0.79(0.56,1.11)$ & $0.64(0.46,0.88)$ & $0.66(0.47,0.92)$ \\
& UK (n=197) & 1.00 & $1.71(0.82,3.59)$ & $2.96(1.26,6.94)$ & $2.31(1.01,5.28)$ & $2.05(0.92,4.54)$ \\
\hline
\end{tabular}

* Derived from 15 multivariable Cox proportional hazards regression analyses (one for each site-stage stratum) adjusted for: age; sex; differentiation; sub-site; socioeconomic (SEIFA) quartile; remoteness of residence; Local Health Network; Medicare Local (see text). Date of censoring of live cases-Dec 31, 2012 
David Roder et al

Table 5. Relative Odds (95\% Confidence Limits) of Adjuvant Therapies for Cancers of the Colon: South Australian Major Public Hospitals, 1980-2010 Cases Treated by Surgery [n=5423] - 3 Multiple Logistic Regression Analyses

\begin{tabular}{|c|c|c|c|c|}
\hline \multicolumn{2}{|l|}{ Groups } & \multirow{2}{*}{$\frac{\text { Radiotherapy }(\mathrm{n}=5423)}{1.00}$} & \multirow{2}{*}{$\frac{\text { Systemic }(n=5423)}{1.00}$} & \multirow{2}{*}{$\frac{\text { Either }(n=5423)}{1.00}$} \\
\hline Age at diagnosis (yrs.): & $<40(n=98)$ & & & \\
\hline & $40-49(n=290)$ & $2.93(0.36,23.7)$ & $1.38(0.75,2.55)$ & $1.50(0.82,2.75)$ \\
\hline & $50-59(n=667)$ & $2.69(0.35,20.7)$ & $1.20(0.67,2.12)$ & $1.28(0.73,2.26)$ \\
\hline & $60-69(n=1283)$ & $2.47(0.33,18.6)$ & $0.72(0.41,1.26)$ & $0.77(0.44,1.33)$ \\
\hline & $70-79(n=1883)$ & $1.79(0.24,13.5)$ & $0.30(0.17,0.53)$ & $0.34(0.19,0.58)$ \\
\hline & $80+(n=1202)$ & $1.00(0.12,8.04)$ & $0.04(0.02,0.07)$ & $0.05(0.03,0.09)$ \\
\hline \multirow[t]{2}{*}{ Sex: } & Male $(n=2685)$ & 1.00 & 1.00 & 1.00 \\
\hline & Female $(n=2738)$ & $0.77(0.51,1.16)$ & $0.93(0.79,1.10)$ & $0.94(0.80,1.10)$ \\
\hline \multirow[t]{5}{*}{ Stage (ACPS): } & $A(n=650)$ & 1.00 & 1.00 & 1.00 \\
\hline & $B(n=1987)$ & $1.51(0.51,4.51)$ & $5.60(3.13,10.0)$ & $5.17(3.00,8.91)$ \\
\hline & $C(n=1512)$ & $2.86(0.99,8.29)$ & $66.3(37.3,117.9)$ & $56.4(32.9,96.8)$ \\
\hline & $\mathrm{D}(\mathrm{n}=1062)$ & $6.97(2.47,19.6)$ & $35.6(19.9,63.5)$ & $33.1(19.2,56.9)$ \\
\hline & UK (n=212) & $6.73(1.81,25.0)$ & $7.99(3.59,17.8)$ & $7.66(3.60,16.3)$ \\
\hline \multirow[t]{4}{*}{ Differentiation: } & Well (n=274) & 1.00 & 1.00 & 1.00 \\
\hline & Moderate $(n=3605)$ & $2.23(0.53,9.37)$ & $1.35(0.85,2.16)$ & $1.45(0.92,2.30)$ \\
\hline & Poor/undiff $(n=1253)$ & $2.23(0.52,9.65)$ & $1.45(0.89,2.35)$ & $1.56(0.97,2.51)$ \\
\hline & $\mathrm{UK}(\mathrm{n}=291)$ & $2.69(0.56,12.9)$ & $0.85(0.48,1.50)$ & $1.01(0.58,1.76)$ \\
\hline \multirow[t]{6}{*}{ Sub-site: } & Proximal $(n=2392)$ & 1.00 & 1.00 & 1.00 \\
\hline & Transverse $(\mathrm{n}=551)$ & $1.36(0.68,2.74)$ & $0.96(0.72,1.27)$ & $0.97(0.73,1.27)$ \\
\hline & Distal $(n=2311)$ & $1.53(0.97,2.39)$ & $0.95(0.79,1.14)$ & $0.98(0.82,1.17)$ \\
\hline & Other/unspec. $(n=169)$ & $1.14(0.39,3.31)$ & $1.05(0.68,1.62)$ & $1.12(0.73,1.71)$ \\
\hline & Rectosigmoid & - & - & - \\
\hline & Rectum & - & - & - \\
\hline \multirow[t]{5}{*}{ Diagnostic period: } & $1980-86(n=608)$ & 1.00 & 1.00 & 1.00 \\
\hline & $1987-94(n=1119)$ & $3.73(1.29,10.8)$ & $3.46(2.14,5.60)$ & $3.43(2.18,5.42)$ \\
\hline & $1995-99(n=1169)$ & $3.37(1.15,9.82)$ & $15.6(9.78,24.8)$ & $13.9(8.92,21.6)$ \\
\hline & $2000-04(n=1120)$ & $4.00(1.38,11.6)$ & $23.0(14.4,36.8)$ & $20.3(13.0,31.6)$ \\
\hline & $2005-10(n=1407)$ & $2.00(0.67,6.00)$ & $27.8(17.5,44.1)$ & $24.2(15.6,37.5)$ \\
\hline
\end{tabular}

Table 6. Relative Odds (95\% Confidence Limits) of Adjuvant Therapies for Cancers of the Rectum: South Australian Major Public Hospitals, 1980-2010 Cases Treated by Surgery [n=2701] - 3 Multiple Logistic Regression Analyses

\begin{tabular}{|c|c|c|c|c|}
\hline Groups & & Radiotherapy $(n=2701)$ & $\begin{array}{c}\text { Treatments } \\
\text { Systemic }(n=2701)\end{array}$ & Either $(n=2701)$ \\
\hline \multirow[t]{6}{*}{ Age at diagnosis (yrs.): } & $<40(\mathrm{n}=56)$ & 1.00 & 1.00 & 1.00 \\
\hline & $40-49(n=161)$ & $0.74(0.36,1.49)$ & $0.92(0.42,1.99)$ & $0.89(0.41,1.92)$ \\
\hline & $50-59(n=415)$ & $0.62(0.32,1.19)$ & $0.59(0.29,1.20)$ & $0.64(0.31,1.29)$ \\
\hline & $60-69(n=794)$ & $0.48(0.25,0.90)$ & $0.51(0.25,1.01)$ & $0.52(0.26,1.04)$ \\
\hline & $70-79(n=828)$ & $0.39(0.21,0.74)$ & $0.28(0.14,0.56)$ & $0.32(0.16,0.64)$ \\
\hline & $80+(n=447)$ & $0.12(0.06,0.25)$ & $0.04(0.02,0.08)$ & $0.07(0.03,0.14)$ \\
\hline \multirow[t]{2}{*}{ Sex: } & Male $(n=1617)$ & 1.00 & 1.00 & 1.00 \\
\hline & Female $(n=1084)$ & $0.80(0.65,0.98)$ & $1.01(0.81,1.25)$ & $0.88(0.72,1.07)$ \\
\hline \multirow[t]{5}{*}{ Stage (ACPS): } & $\mathrm{A}(\mathrm{n}=501)$ & 1.00 & 1.00 & 1.00 \\
\hline & $B(n=780)$ & $3.08(2.15,4.40)$ & $3.89(2.63,5.76)$ & $3.71(2.62,5.24)$ \\
\hline & $C(n=831)$ & $6.95(4.91,9.82)$ & $23.5(15.9,34.7)$ & $19.5(13.7,27.6)$ \\
\hline & $D(n=474)$ & $3.22(2.20,4.73)$ & $9.50(6.33,14.3)$ & $9.90(6.84,14.3)$ \\
\hline & $\mathrm{UK}(\mathrm{n}=115)$ & $2.22(1.13,4.37)$ & $1.87(0.82,4.29)$ & $3.29(1.72,6.27)$ \\
\hline \multirow[t]{4}{*}{ Differentiation: } & Well $(n=126)$ & 1.00 & 1.00 & 1.00 \\
\hline & Moderate $(n=2020)$ & $0.90(0.53,1.52)$ & $1.50(0.83,2.73)$ & $1.16(0.68,1.98)$ \\
\hline & Poor/undiff $(n=407)$ & $1.06(0.60,1.88)$ & $1.30(0.69,2.47)$ & $1.28(0.72,2.27)$ \\
\hline & $\mathrm{UK}(\mathrm{n}=148)$ & $1.12(0.59,2.13)$ & $1.57(0.77,3.22)$ & $1.18(0.61,2.29)$ \\
\hline \multirow[t]{6}{*}{ Sub-site: } & Proximal & - & - & - \\
\hline & Transverse & - & - & - \\
\hline & Distal & - & - & - \\
\hline & Other/unspec. & - & - & - \\
\hline & Rectosigmoid $(n=767)$ & 1.00 & 1.00 & 1.00 \\
\hline & Rectum $(n=1934)$ & $3.47(2.69,4.48)$ & $1.31(1.04,1.65)$ & $1.72(1.38,2.14)$ \\
\hline \multirow[t]{5}{*}{ Diagnostic period: } & $1980-86(n=320)$ & 1.00 & 1.00 & 1.00 \\
\hline & $1987-94(n=621)$ & $4.15(2.29,7.52)$ & $15.2(6.02,38.4)$ & $6.66(3.79,11.7)$ \\
\hline & $1995-99(n=583)$ & $8.48(4.73,15.2)$ & $45.3(18.0,113.9)$ & $17.4(9.93,30.6)$ \\
\hline & $2000-04(n=563)$ & $13.7(7.65,24.6)$ & $82.3(32.7,207.6)$ & $31.8(18.0,56.0)$ \\
\hline & $2005-10(n=614)$ & $15.1(8.45,26.9)$ & $116.1(46.0,292.7)$ & $42.9(24.3,75.6)$ \\
\hline
\end{tabular}


Table 7. Relative Odds (95\% Confidence Limits) of Adjuvant Therapies for Colon and Rectal Cancers by Stage (ACPS) and Diagnostic Period: South Australian Major Public Hospitals, 1980-2010 Cases Treated by Surgery*

\begin{tabular}{lccccc}
\hline Site \& Stage & $1980-86$ & $1987-94$ & $1995-99$ & $2000-04$ & $2005-10$ \\
\hline Colon & & & & & \\
A (n=650) & - & 1.00 & $0.37(0.04,3.04)$ & $0.97(0.20,4.63)$ & - \\
B (n=1987) & 1.00 & $2.43(0.68,8.71)$ & $8.15(2.44,27.2)$ & $16.1(4.86,52.9)$ & $17.0(5.19,55.8)$ \\
C (n=1512) & 1.00 & $9.93(4.16,23.7)$ & $52.6(22.0,125.7)$ & $83.6(34.5,202.5)$ & $68.9(29.0,163.9)$ \\
D (n=1062) & 1.00 & $1.62(0.81,3.27)$ & $5.47(2.86,10.5)$ & $7.35(3.84,14.1)$ & $13.1(6.9,25.2)$ \\
UK (n=212) & 1.00 & $0.55(0.08,3.90)$ & $1.05(0.11,10.3)$ & $0.63(0.04,11.2)$ & $1.41(0.15,13.3)$ \\
Rectum & & & & & \\
A (n=501) & 1.00 & $4.70(0.57,39.6)$ & $3.55(0.42,29.9)$ & $10.1(1.26,81.0)$ & $21.5(2.79,166.1)$ \\
B (n=780) & 1.00 & $7.16(1.62,31.7)$ & $17.3(4.00,74.5)$ & $45.1(10.5,194.3)$ & $61.6(14.3,264.8)$ \\
C (n=831) & 1.00 & $9.19(4.07,20.8)$ & $36.4(16.9,92.2)$ & $55.0(23.1,131.1)$ & $48.7(21.1,112.7)$ \\
D (n=474) & 1.00 & $5.41(1.50,19.6)$ & $12.8(3.62,45.6)$ & $24.3(6.86,86.3)$ & $38.8(10.6,142.1)$ \\
UK (n=115) & 1.00 & $1.50(0.24,9.50)$ & $8.11(0.85,77.7)$ & $5.70(0.76,42.5)$ & $2.89(0.34,24.5)$ \\
\hline
\end{tabular}

*Derived from 10 logistic regression analyses (one for each site and stage stratum), adjusting for age; sex; differentiation; sub-site (see text)

hemicolectomies increasing from $50.4 \%$ in $1980-1986$ to $56.4 \%$ in 2005-2010; (2) left hemicolectomies decreasing from $9.7 \%$ in $1980-1986$ to $4.1 \%$ in $2005-2010$; and (3) partial colectomies decreasing from $4.6 \%$ in $1980-1986$ to $1.2 \%$ in $2005-2010$.

The most common surgical procedures for rectal cases were anterior resections $(59.0 \%)$, followed by abdominoperineal resections $(21.8 \%)$, Hartmann resections $(8.2 \%)$ and other or unknown procedures $(10.9 \%)$. The distribution varied by period ( $\mathrm{p}<0.001)$, with the proportion that were: $(i)$ anterior resections increasing from $53.3 \%$ in $1980-1986$ to $64.3 \%$ in $2005-2010$; and (ii) abdominoperineal resection decreasing from $31.5 \%$ in $1980-1986$ to $18.7 \%$ for $2000-2010$, during which time no further downward trend was suggested.

\section{Adjuvant therapies (surgical cases)}

Bi-variable analyses: Of the 8,064 surgical cases, $2,292(28.4 \%)$ had adjuvant radiotherapy and/or systemic therapy. This percentage increased from $4.5 \%$ for 1980 1986 to $40.8 \%$ in $2005-2010$ for all stages collectively. It was higher for rectal cases at $36.9 \%$ (increasing from $5.0 \%$ in 1980-1986 to $56.0 \%$ in 2005-2010) compared with $23.9 \%$ for colonic cases (increasing from $4.3 \%$ in 1980 1986 to $34.1 \%$ for $2005-2010$ ). Notably the proportion of colorectal cases having adjuvant therapy was highest for stage C, increasing from $5.1 \%$ in $1980-1986$ to $62.6 \%$ in 2005-2010.

Meanwhile the percentage receiving adjuvant radiotherapy was higher for rectal cases at $24.5 \%$ (increasing from $4.4 \%$ in $1980-1986$ to $36.5 \%$ for 2005 2010 for all stages and from $7.5 \%$ in $1980-1986$ to $60.2 \%$ for stage $\mathrm{C}$ ) than the $1.9 \%$ for colonic cases (increasing from $0.7 \%$ in $1980-1986$ to $1.4 \%$ for $2005-2010$, after reaching a peak of $2.5 \%$ in 2000-2004).

Chemotherapy (or other systemic therapy) was provided to $25.7 \%$ of colorectal cases, with this percentage increasing from $3.0 \%$ in $1980-1986$ to $38.4 \%$ in 2005 2010 for all stages combined and from $2.6 \%$ in 1980 1986 to $64.7 \%$ in $2005-2010$ for stage C. The percentage receiving chemotherapy or other systemic therapy was higher for rectal cases at $31.1 \%$ (increasing from $1.6 \%$ in $1980-1986$ to $50.0 \%$ for $2005-2010$ ) than the $23.0 \%$ for colonic cases (increasing from $3.8 \%$ in 1980-1986 to $33.3 \%$ in 2005-2010).

Multivariable analyses: Multiple logistic regression analyses for colon cancer (Table 5) showed that predictors of treatment types were: ( $i$ ) Radiotherapy: (1) diagnosis in 1987-2004 compared with the 1980-1986 reference period; and (2) more advanced stage; (ii) Systemic therapy: (1) younger age; (2) more recent diagnostic period; and (3) stage C and (less so) stages D and B than stage A; (iii) Either radiotherapy or systemic therapy: (1) younger age, with a comparatively low utilization applying for ages over 70 years; (2) more recent diagnostic period; and (3) stage C and (less so) stages D and B than stage A.

Multiple regression analyses for rectal cancer (Table $6)$ indicated that predictors of treatment types were: $(i)$ Radiotherapy: (1) younger age; (2) male sex; (3) stage $\mathrm{C}$ and (less so) stage $\mathrm{D}$ and $\mathrm{B}$ compared with stage A; (4) rectum compared with rectosigmoid sub-site; and (5) more recent diagnostic period; (ii) Systemic therapy: (1) younger age, with comparatively low utilization applying for ages over 70 years; (2) stage C and (less so) stage D and $\mathrm{B}$ compared with stage $\mathrm{A}$; (3) rectum compared with rectosigmoid sub-site; and (4) more recent diagnostic period; (iii) Either radiotherapy or systemic therapy: (1) younger age; (2) stage C and (less so) stage D and $\mathrm{B}$ compared with stage A; (3) rectum compared with rectosigmoid sub-site; and (4) more recent diagnostic period.

Multiple logistic regression analyses showed, after adjusting for age, sex, differentiation, and sub-site, that there were upward trends in the use of adjuvant (including first-round treatments with palliative intent) for stages B- D colonic cases and rectal cases of all stages (Table 7).

In particular, an upward trend for colonic cases applied to chemotherapy (or other systemic therapy) for stage $\mathrm{C}$ and less so, stages $\mathrm{B}$ and $\mathrm{D}$, where the relative odds for 2005-2010 compared with 1980-86 were 105.9 (37.6, 298.5), 25.9 (6.2,108.7) and $12.2(6.4,23.3)$ respectively. Multiple logistic regression analyses also showed, after adjusting for age, sex, differentiation, and sub-site, that a pronounced upward trend applied for rectal cancer in systemic therapy for stage $\mathrm{C}$ and (less so) stage $\mathrm{D}$, where the relative odds for 2005-2010 compared with 
David Roder et al

1980-86 were $102.4(30.2,346.9)$ and $42.1(9.3,191.0)$ respectively.

Upward trends in the use of radiotherapy for rectal cases applied to all stages, such that the relative odds of radiotherapy for 2005-2010 compared with 1980-1986 were: stage A: $17.5(2.3,135.5)$; stage B: $40.4(9.4,174.3)$; stage C: 10.7 (4.6, 24.7); and stage D: $12.9(2.9,58.5)$.

Consistent with best-practice guidelines, systemic therapy use evolved from single-agent 5FU (administered to $80 \%$ of chemotherapy patients in 1980-86) to 5FU with leucovorin (administered to $71 \%$ of chemotherapy patients in 1995-1999). By 2005-2010, the range of therapies had broadened, for colonic cases to include FOLFOX (with or without bevacizumab) and capecitabine (with or without oxaliplatin). Meanwhile protracted infusion of 5FU remained a common chemotherapy for rectal cases in association with radiotherapy.

\section{Discussion}

Since 1980-1986, when the five-year survival from colorectal cancer was $48 \%$ at these tertiary hospitals, a progressive increase occurred to $63 \%$ five-year survival in 2005-2010. This compares with a five-year relative survival (period method) for all colorectal cancers in Australia for 1982-87 of 48\% and for 2006-2010 of 66\% (AIHW, 2012). Considering the potential for slightly higher survival estimates from period than cohort methods, survivals at these tertiary hospitals appeared broadly similar to those for Australia overall.

This similarity in survival is interpreted favourably for these referral centres, given more advanced stage distributions at diagnosis. Stages of all colorectal cases aged 55-74 years diagnosed in South Australia in 20032008 were found in a study by Cole et al. (2013) to be: A-23\%; B-33\%; C-30\%; and D-14\%, whereas a more advanced stage distribution applied to cases of equivalent age and diagnostic years at the present tertiary hospitals of: A-15\%; B-30\%; C-29\%; and D-25\%.

Without national data on stage, potential contributions to overall increases in survival of trends in stage and stagespecific survival are difficult to estimate. However, U.S.A. SEER data show a similar increase in five-year period survival, peaking at $66 \%$ for 2004-2010 diagnoses as for Australia (AIHW, 2012; Howlader, 2014), but with little evidence of a trend towards earlier stage (Howlader, 2014) [note: the stage distribution of SEER cases in 1983-1990 was localized-39\%, regional-40\%, and distant- $20 \%$ (Ries, 1994), which was similar to the 2004-2010 distribution of localized-42\%, regional-38\%, and distant $21 \%$.

A key finding of the present study was the approximate halving of the hazards ratio (case fatality) between 198086 and 2005-2010 after adjusting for stage, differentiation, sub-site, age, sex, and postcode-derived socioeconomic status, remoteness and Local Health Network and Medicare Local area.(ABS, 1998). This indicates appreciable survival gains at these hospitals that are not attributable to improvements in stage.

As for the SEER data, a trend towards earlier stage at diagnosis was not observed. On the contrary, there was a temporal trend towards more advanced stages
( $p=0.007$ ), with the percentage of cases with distant metastases increasing from $22 \%$ in $1980-1999$ to $26 \%$ in 2000-2010. The extent to which this reflects patient selection factors, with an increased tendency for more advanced cases to attend SACCR hospitals, as opposed to increased surveillance intensity or advances in diagnostic sensitivity, is not known.

It is plausible that improved imaging would have increased diagnostic sensitivity, leading to stage shift and potentially an artificial contribution to improvements in stage-specific survival. However, it is unlikely that this would account for a halving of the disease-specific hazards ratio. It is evident from Table 1 that for changes in survival between 1980-1986 and 2005-2010 to have been an artificial change (i.e., for colorectal death outcomes for these cases to have remained unchanged), dates of diagnosis would need to have been brought forward on average by more than three years per case, which seems implausible.

Population-based data also suggest that the halving of the hazards ratio in the present study is real, in that population-based age-standardized colorectal cancer mortality rates reduced in Australia by $44 \%$ between 1980 1986 and 2005-2010, with a corresponding age-standardi zed incidence increase of 6\% (AIHW, 2013). This is consistent with a $47 \%$ reduction in numbers of colorectal cancer deaths per 100 colorectal cancer cases, which is broadly consistent with the halving of the disease-specific hazards ratio observed in SACCR cases. These trends point to real and substantial survival gains, although it is still acknowledged the potential for a small artificial contribution from stage shift existed in the present study.

When separate models were applied for each stage of colon and rectal cancer, with adjustment for age, sex, differentiation and sub-site, they all showed reductions in hazards ratios, although the trend was not statistically significant for stage A colon cancer despite a low hazards ratio of 0.46 for 2005-2010 compared with 1980-86 (not significant due to low statistical power from small numbers of deaths). Apart from stage A cases, where estimates were imprecise, the largest reductions in hazards ratios applied to stage $\mathrm{C}$ for both colonic and rectal cancers.

Statistical associations of survivals with stage, differentiation, sub-site and age at diagnosis were consistent with results of an earlier study at these hospitals (Luke et al., 2005). No association was found of survival with sex and residential area derived socioeconomic status, remoteness, or Local Health Network or Medicare Local area, which is encouraging and suggestive of a reasonable equity in service provision and outcomes at these hospitals.

The lower disease-specific survivals for old patients aged 80 years and over have been reported before for colorectal and many other cancers (AIHW, 2012). Surgical cases aged 80 years and over had lower relative odds of: systemic therapies for colonic cancers; and adjuvant radiotherapy and systemic therapies for rectal cancers. The extent to which lower exposure to adjuvant therapies explains the lower survivals observed in these old patients is not known. Older patients are more likely to have comorbidity and more general frailty which could reduce 
their resilience to active therapy, such that compromises need to be made to treatment protocols.

Treatment of surgical cases with adjuvant therapies has increased significantly over time, which is likely to have contributed to the increases in survivals (U.S. Department of Health and Human Services, 1990; NHMRC, 1999; Australian Cancer Network Colorectal Cancer Guidelines Revision Committee, 2005; Schmoll et al., 2012). The pattern of increase corresponds with evidence-based treatment guidelines, insofar as the increase in radiotherapy was greater for rectal cancers and the increases in adjuvant systemic therapy more pronounced for stage $\mathrm{C}$, both for colonic and rectal cancers (U.S. Department of Health and Human Services, 1990; NHMRC, 1999; Australian Cancer Network Colorectal Cancer Guidelines Revision Committee, 2005; Schmoll et al., 2012).

The percentage of stage $\mathrm{C}$ colorectal cases less than 70 years of age having systemic therapy as part of their primary treatment increased to $81 \%$ during 1995-2010. Based on the ratio of those treated to those offered this care, as indicated by national survey data (NCCI, 2000), it seems that virtually all these younger patients would have been offered adjuvant systemic treatment. Meanwhile the percentage of stage $\mathrm{C}$ rectal cases less than 70 years of age having radiotherapy as part of their primary treatment increased to $63 \%$ by 2005-2010. Again, based on the ratio of those treated to those offered radiotherapy, as indicated by national survey data (NCCI, 2000), it would appear that about $80 \%$ of all these younger patients may have been offered radiotherapy.

Reasons for the sub-optimum uptake of radiotherapy for rectal cases require further investigation, including the potential for a lower uptake for stage A, females, and potentially for cases with increased co-morbidity and frailty in the older age ranges from age 60 years. About a quarter of cases resided in non-metropolitan areas and the potential for access barriers in the more remote of these areas exists, given the location of radiotherapy centres in metropolitan areas.

Apart from increases in use of adjuvant therapies over time, surgical sub-specialization has increased and improvements in surgical management and technique have occurred, including for example, the increased use of total mesorectal excisions (Luke et al., 2005). In addition, increased use of neo-adjuvant therapy would have increased the potential for complete resection for some cancers otherwise not able to be excised. There is also evidence that increased resection of hepatic and pulmonary metastases may be extending survivals (Neo et al., 2011; Due et al., 2012; Padman et al., 2013).

This study illustrates the value of clinical cancer registries in Australia for monitoring differences in patterns of care and survival by stage (Neo et al., 2011; Due et al., 2012; Padman et al., 2013), and for providing complementary information data to population-based registry data (Coleman et al., 2011; Neo et al., 2011; Due et al., 2012; Padman et al., 2013; Beckmann et al., 2014b; Roder, 2014; Roder et al., 2014; Taheri et al., 2014; Roder, 2015). The electronic transfer of prognostic data from structured pathology reporting, and continuous updating of this reporting to account for new scientific evidence, will ensure that clinical registry data remain effective for assessing health-system performance (Roder et al., 2014; Roder, 2015).

Clinical registries augment linked population-based registry and administrative treatment data with data of greater detail and quality that can be used: $(i)$ to validate courser population-based evidence for those patient groups where the data systems overlap; (ii) to monitor health-system performance; (iii) to undertake clinical research, including $\mathrm{T} 1$ translation of research from laboratory to clinical settings; (iv) to undertake comparative effectiveness research; and (v) to check how outcomes from clinical trials translate to outcomes in routine practice, including outcomes in older patients with multiple and complex disease who may not have been included in the trials (Beckmann et al., 2014a; Roder et al., 2014; Roder, 2015). The value of clinical and population-based registries can be greatly increased when data extracts from these registries are linked, along with linkage to screening registries and other administrative datasets (Beckmann et al., 2014a; Roder et al., 2014; Roder, 2015).

In conclusion: $(i)$ There have been pronounced increases in survivals from colorectal cancer at major public hospitals in South Australia due to increases in stage-specific survivals; (ii) Use of adjuvant therapies has increased substantially at these hospitals and the pattern of change in therapy accords with clinical guideline recommendations; (iii) Reasons for sub-optimal use of radiotherapy for rectal cases warrants further investigation, including the potential for remoteness of residence to impede uptake in metropolitan-based centres.

\section{Acknowledgements}

This study received funding support from the Royal Adelaide Hospital Health Services Charitable Gift Board, Cancer Council South Australia, the South Australia Health and Medical Research Institute, University of South Australia, and the South Australian Department of Health. This funding support is gratefully acknowledged.

\section{References}

(ABS) Australian Bureau of Statistics (1998). 1996 census of population and housing. Socio-economic indexes for areas, ABS, Canberra.

(AIHW \& AACR) Australian Institute of Health and Welfare \& Australasian Association of Cancer Registries (2001). Cancer survival in Australia. Part 2: statistical tables, Cancer Series, vol. 19 Cat. No. CAN14, Canberra.

(AIHW) Australian Institute of Health and Welfare (2012). Cancer survival and prevalence in Australia: period estimates from 1982 to 2010, cancer series No. 69. Cat. No. CAN 65, AIHW, Canberra.

(AIHW) Australian Institute of Health and Welfare (2013). Australian cancer incidence and mortality (ACIM) books, AIHW, Canberra. <available at: http://www.aihw.gov.au/ acim-books/>

Armitage P, Berry, G (eds.) (1987). Statistical Methods in Medical Research, Blackwell Scientific Publications, 
Oxford.

Australian Cancer Network Colorectal Cancer Guidelines Revision Committee (2005). Guidelines for the prevention, early detection and management of colorectal cancer. Cancer Council Australia \& Australian Cancer Network, Sydney.

Australian Health Technology Advisory Committee (1997). Colorectal cancer screening, Commonwealth Department of Health and Family Services, Canberra.

Beckmann KR, Bennett A, Young GP, et al (2014a). Treatment patterns among colorectal cancer patients in South Australia: a demonstration of the utility of population-based data linkage. J Eval Clin Pract, 20, 467-77.

Beckmann KR, Roder DM, Hiller JE, et al (2014b). Influence of mammographic screening on breast cancer incidence trends in South Australia. Asian Pac J Cancer Prev, 15, 3105-12.

Bonett A, Roder D, Esterman A (1988). Cancer case-survival rates for South Australia: a comparison with US rates and a preliminary investigation of time trends. Med J Aust, 148, 556-9.

Cole SR, Tucker GR, Osborne JM, et al (2013). Shift to earlier stage at diagnosis as a consequence of the national bowel cancer screening program. Med J Aust, 198, 327-30.

Coleman MP, Forman D, Bryant H, et al (2011). Cancer survival in Australia, Canada, Denmark, Norway, Sweden, and the UK, 1995-2007 (the international cancer benchmarking partnership): an analysis of population-based cancer registry data. Lancet, 377, 127-38.

Department of Health \& (AIHW) Australian Institute of Health and Welfare (2008). National bowel screening program monitoring report, Canberra, AIHW, Cancer Series 44. Cat. no. 40

Due SL, Wattchow DA, Sweeney JL, et al (2012). Colorectal cancer surgery 2000-2008: evaluation of a prospective database. ANZ J Surg, 82, 412-9.

Ferlay J, Soerjomataram I, Ervik M, et al (2013), Cancer incidence and mortality worldwide: IARC CancerBase No.11. Lyon, France: IARC <available at: http://globocan. iarc.fr>.

Hardcastle JD, Chamberlain JO, Robinson MH, et al (1996). Randomised controlled trial of faecal-occult-blood screening for colorectal cancer. Lancet, 348, 1472-7.

Hewitson P, Glasziou P, Watson E, et al (2008). Cochrane systematic review of colorectal cancer screening using the fecal occult blood test (hemoccult): an update. Am J Gastroenterol, 103, 1541-9.

Howlader N, Noone AM, Krapcho M, et al (2014). SEER cancer statistics review, 1975-2011, national cancer institute, Bethesda, MD.

Jong KE, Smith DP, Yu XQ, et al (2004). Remoteness of residence and survival from cancer in New South Wales. Med J Aust, 180, 618-22.

Jorgensen OD, Kronborg O, Fenger C (2002). A randomised study of screening for colorectal cancer using faecal occult blood testing: results after 13 years and seven biennial screening rounds. Gut, 50, 29-32.

Kronborg O, Fenger C, Olsen J, et al (1996). Randomised study of screening for colorectal cancer with faecal-occult-blood test. Lancet, 348, 1467-71.

Kronborg O, Fenger C, Sondergaard O, et al (1987). Initial mass screening for colorectal cancer with fecal occult blood test. A prospective randomized study at Funen in Denmark. Scand J Gastroenterol, 22, 677-86.

Luke CG, Koczwara B, Moore JE, et al (2005). Treatment and survival from colorectal cancer: the experience of patients at South Australian teaching hospitals between 1980 and 2002. Clin Oncol (R Coll Radiol), 17, 372-81.

Mandel JS, Church TR, Bond JH, et al (2000). The effect of fecal occult-blood screening on the incidence of colorectal cancer. $N$ Engl J Med, 343, 1603-7.

(NCCI) National Cancer Control Initiative (2000). The national colorectal cancer care survey. Australian clinical practice in 2000, Clinical Governance Unit, Melbourne.

Neo EL, Beeke C, Price T, et al (2011). South Australian clinical registry for metastatic colorectal cancer. ANZ J Surg, 81, 352-7.

(NHMRC) National Health and Medical Research Council (1999). Clinical practice guidelines. prevention, early detection and management of colorectal cancer, Commonwealth of Australia, Canberra.

Padman S, Padbury R, Beeke C, et al (2013). Liver only metastatic disease in patients with metastatic colorectal cancer: impact of surgery and chemotherapy. Acta Oncol, 52, 1699-706.

Ries LAG, Miller BA, Hankey BF, et al (1994). SEER Cancer Statistics Review, 1973-1991, National Cancer Institute, Bethesda, MD.

Roder D (2014). What roles should population-based cancer registries be playing in the $21^{\text {st }}$ century? Reflections on the Asian Cancer Registry Forum, Bangkok, February 2014. Asian Pac J Cancer Prev, 15, 1895-6.

Roder D, Buckley E (2015). Translation from clinical trials to routine practice: How to demonstrate community benefit. Asia Pac J Clin Oncol, [Epub ahead of print].

Roder DM, Fong KM, Brown MP, et al (2014). Realising opportunities for evidence-based cancer service delivery and research: linking cancer registry and administrative data in Australia. Eur J Cancer Care, 23, 721-7.

(SACR) South Australian Cancer Registry (1999). Epidemiology of Cancer in South Australia. Incidence, mortality, and survival 1977 to 1998 . Incidence and mortality 1998, analysed by type and geographical location. Epidemiology Branch, Department of Health, Adelaide.

(SACR) South Australian Cancer Registry (2007). Cancer in South Australia 2004, with incidence projections to 2007, epidemiology branch, department of health, Adelaide.

Schmoll HJ, Van Cutsem E, Stein A, et al (2012). ESMO consensus guidelines for management of patients with colon and rectal cancer. a personalized approach to clinical decision making. Ann Oncol, 23, 2479-516.

Scholefield JH, Moss S, Sufi F, et al (2002). Effect of faecal occult blood screening on mortality from colorectal cancer: results from a randomised controlled trial. Gut, 50, 840-4.

STATA (2012). Data analysis and statistical software, Ver. 12, StataCorp LP, college station, Texas.

Taheri N, Fazel A, Mahmoodzadeh H, et al (2014). Epidemiology of female reproductive cancers in Iran: results of the Gholestan population-based cancer registry. Asian Pac J Cancer Prev, 15, 8779-82.

U.S. Department of Health and Human Services, National Institutes of Health (NIH) (1990). NIH Consensus Development Program: Adjuvant Therapy for Patients with Colon and Rectum Cancer, NIH Consensus Statement Online. April 16-18 1990, 1-25. <available at: http:// consensus.nih.gov/1990/1990adjuvanttherapycolonrectalc ancer079html.htm> 